Organ, Cybernetics, Artificial Intelligence, Cyborg, Transpecies Society, Method, Senses, Prosthesis. Afanador, Tatiana and Judit Parés. 2019. "Designing Organs at the Transpecies Society: Hybrid Practices between Cybernetics and Artificial Intelligence". Temes de Disseny 35: 140-153.

\title{
Designing Organs at the
} Transpecies Society: Hybrid Practices between Cybernetics and Artificial Intelligence
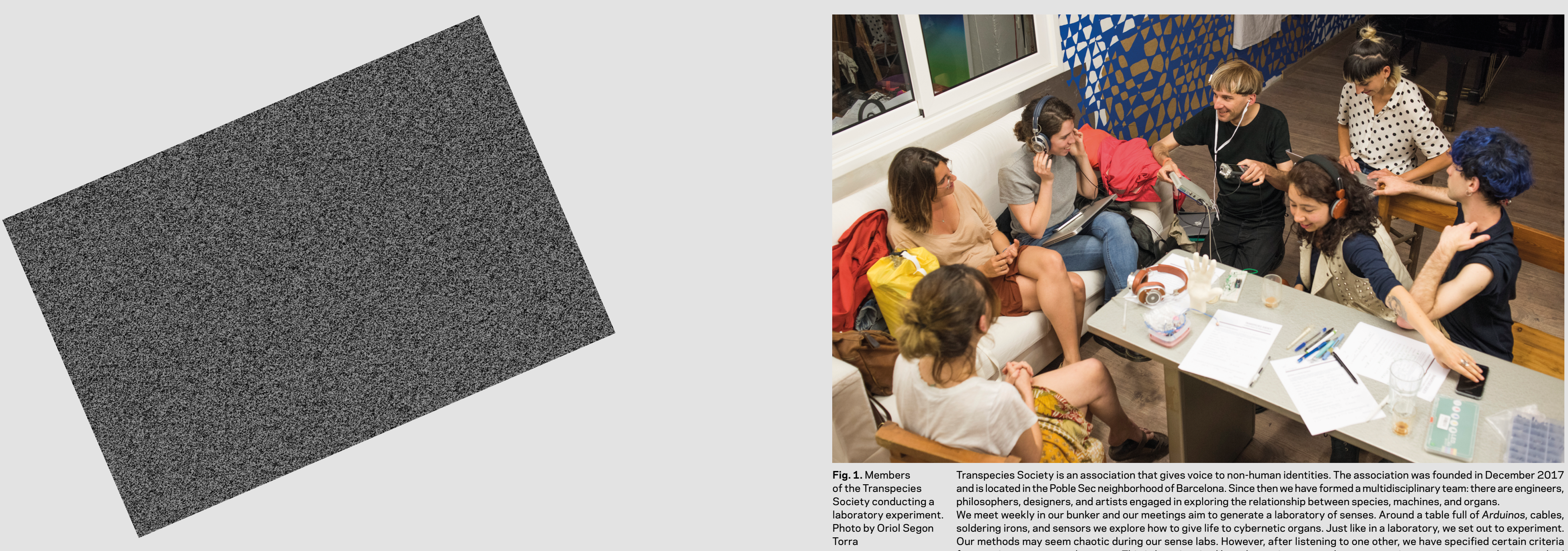

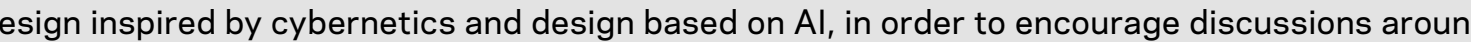

The design of cybernetic organs does not have many current references from which to seek inspiration. However, we think that Transpecies Society members are tinkering with ambiguities about what is natural and what is artificial, incorporating these organs in order to reveal new environmental because being there places us within today's technology.

\section{Fig. 1. Members
of the Transpecics}

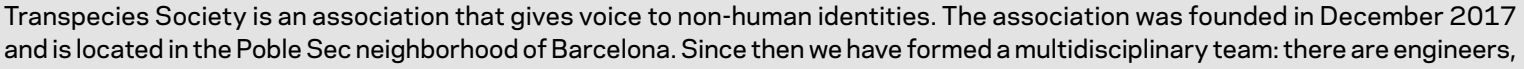
Society conducting a
laboratory experiment

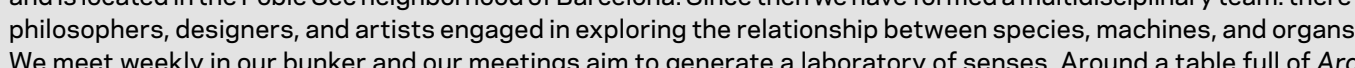




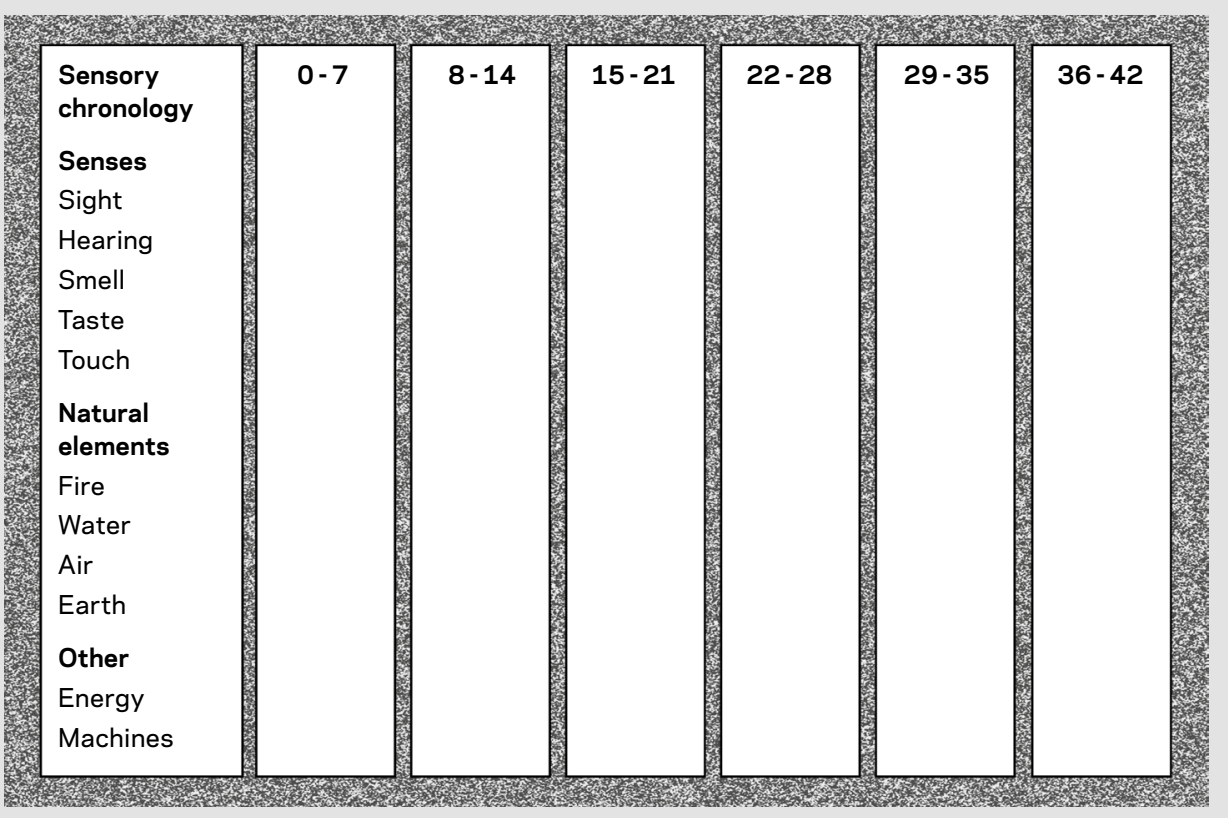

Fig. 3. Data sheet of the senses

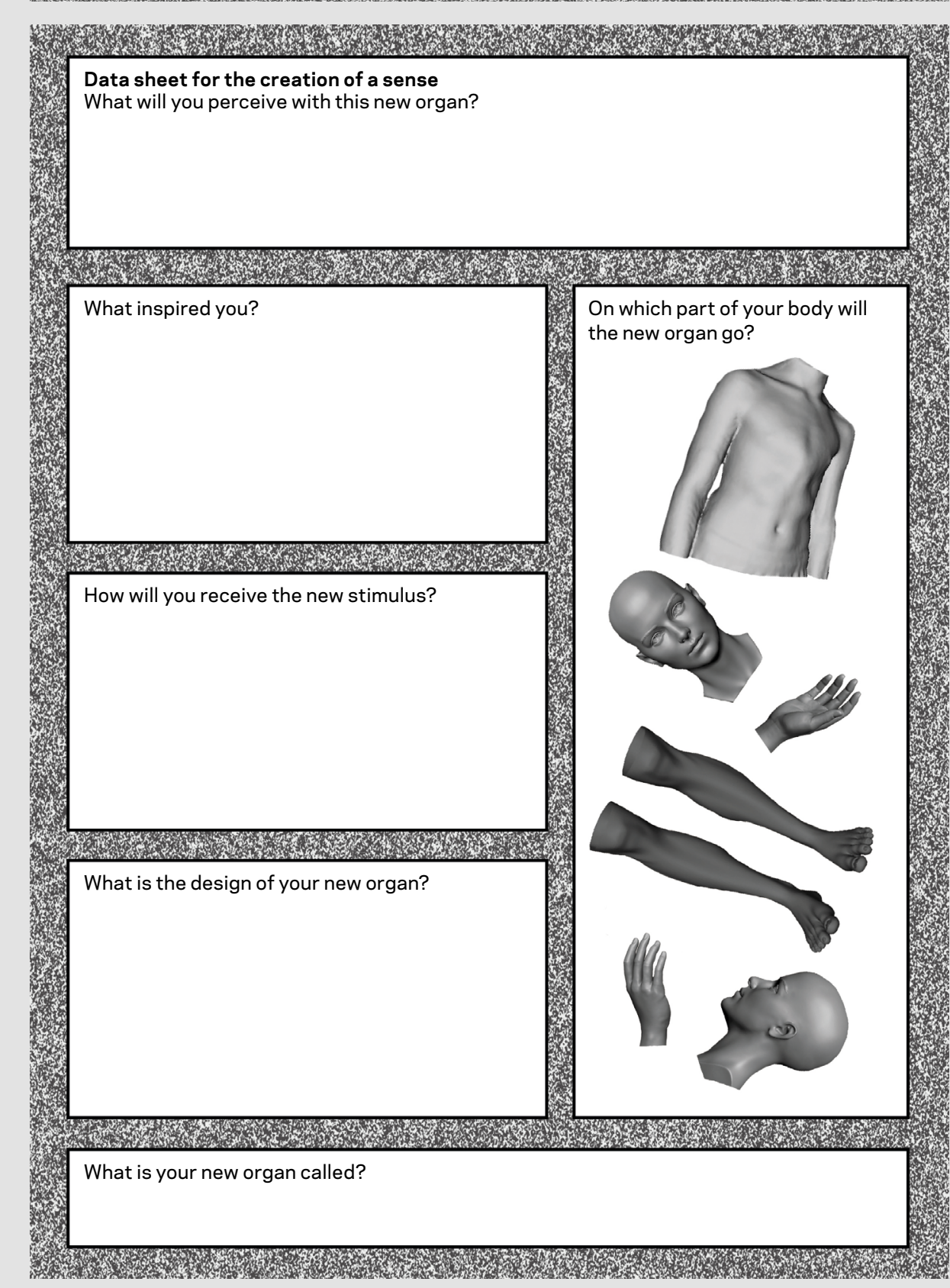

TEMES DE DISSENY \#35

PICTORIAL

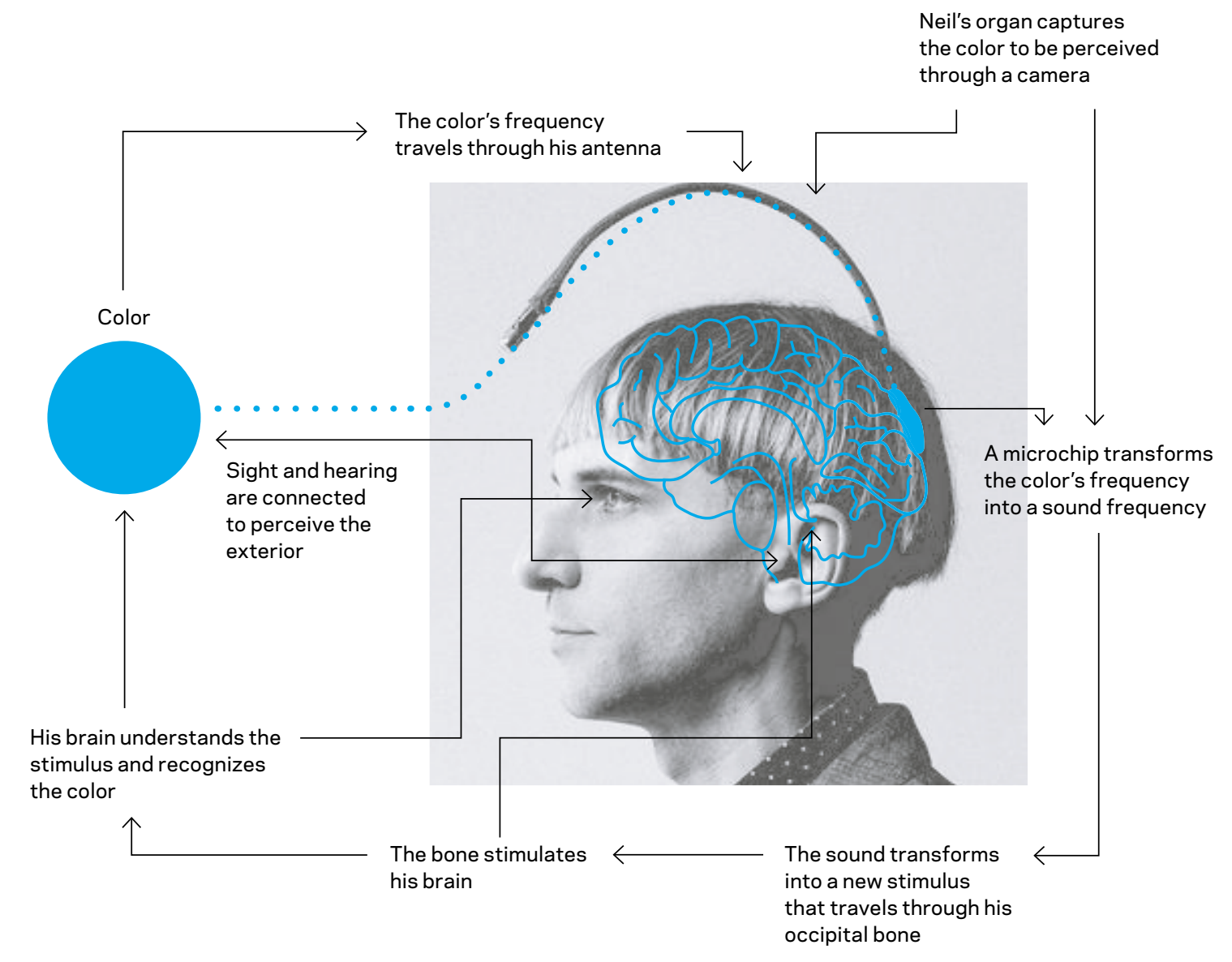

We consider cyborgs [1] those who wish to create cybernetic organs to add a Fig. 4. Representation of Noil Harbisson's organ, inspired by the concept of new sense or input. The path that runs through this hybrid between machine cybernetic feedback

and organ begins with a prior introspection that we call sensory chronology

chronological axis divided into seven-year periods. In this chronology, everyone analyzes the importance of the four elements of nature in relation to their own biological senses to find repetitive patterns or relevant perceptions in their ilife experied by supernatura artifacts as part of this chronology.

The second phase consists of a data sheet in which the where, how, what, and why of the sense is answered. Once the sense is defined, we proceed with its into new software and hardware. The Transpecies Society is a design examp in which other non-human species and all of nature are taken into account. Thus, software for a cybernetic body is not limited to the creation of a binary code. with the body the brain and the the sense of Kai- This sense will show us, through sounds, the existence of subatomic particles called muons. The movements of the muons will be coded to compose a piece of music. Thus, Kai's body will no longer be indifferent to Cyberntic design is approaching attistic practic res (Mur

one of the main reasons for this proximity is that the dest creating an organ is the composition of a network in which various types of organizations are combined (circular causality or feedback) [2].
For example, the structure of the network that composes

or ex Harbisson's body convert into sounds. Neil's sonochromatic sense was invented so he could feel an input generated from the feedback between color and sound in the head and perceived by a cybernetic organ that is at the seeme timated eye and an ear.

T. AFANADOR / J. PARÉS

143 


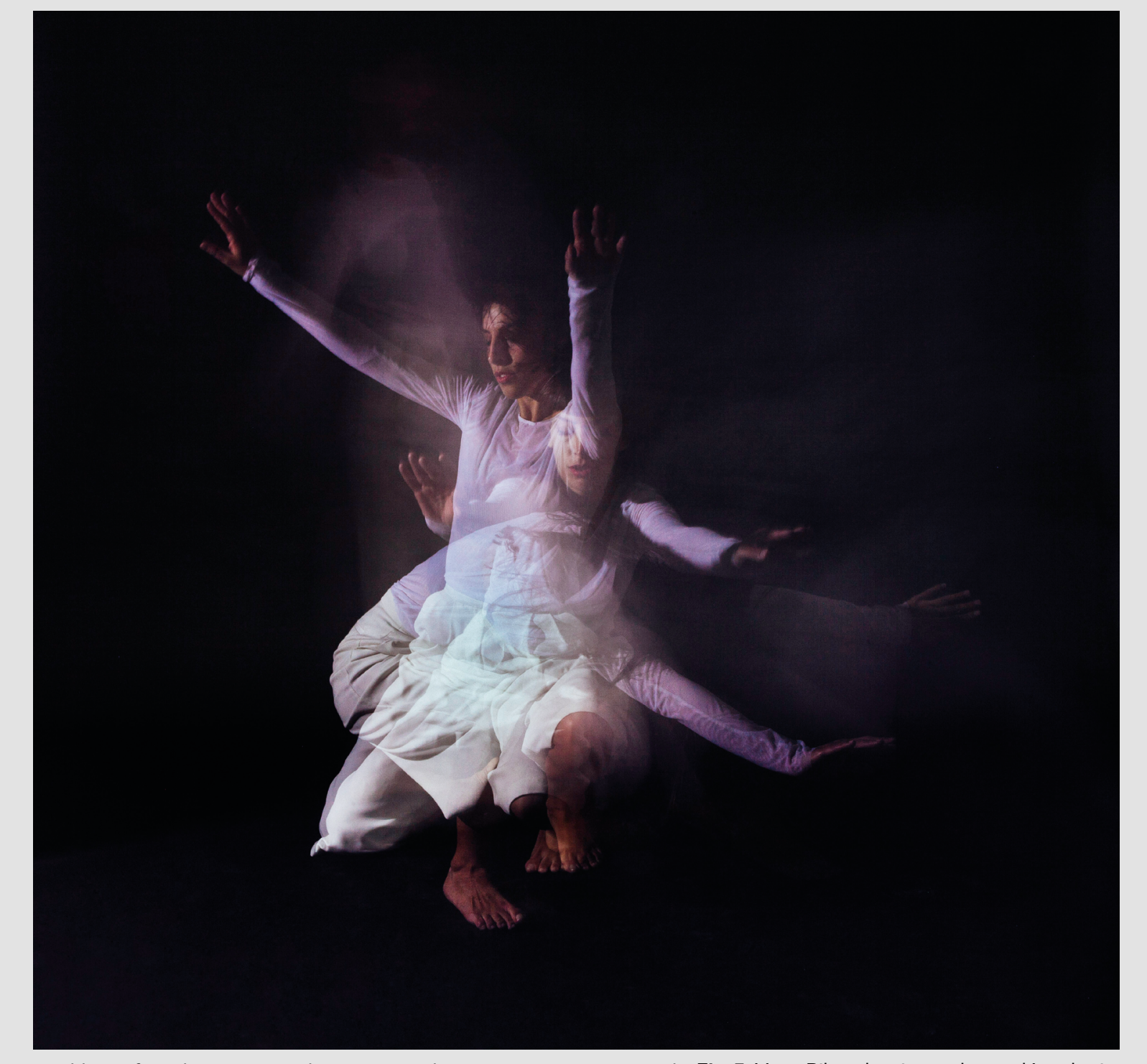

In addition, for cyberneticians, the interaction between various organs and
machines. 5 . Moon Ribas dancing to the earth's pulse inilar to the interaction between colors in a pictorial work: just her performance Waiting for Earthquakes. machines is similar to the interaction between colors in a pictorial work: just her performance Waiting
as the entire landscape of a painting is altered by changing a hue of yellow, Photo by Kathy Anne Lim

the input received by an organ is modified if the part of the body where it is

located is changed. When looking at Moon Ribas's seismic sensor, we see

how cyborgs are weaving the synergy between cybernetics and art. In the
performance called Waiting for Earthquakes, an artwork and public expres mevements are a language that expresses the output generated by a seism sensor implanted in her feet.

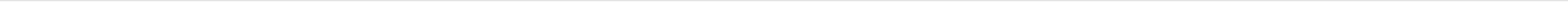




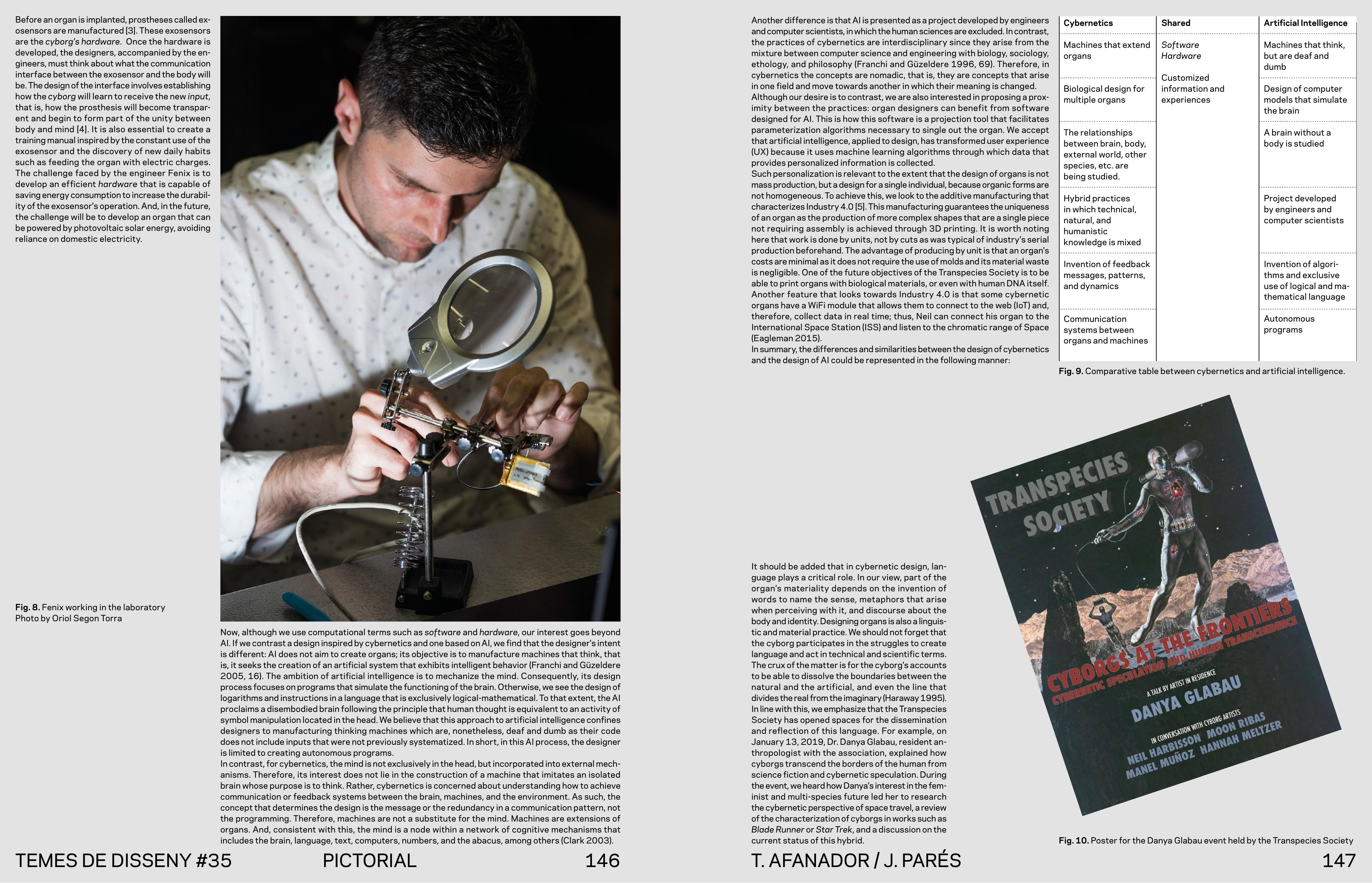


ENDNOTES

tand what a cyborg is, we refer to the original definition of Cyborg

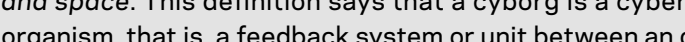
and a machine (Clynes and Kline 1960, 27). However, it should be noted that the challenge of making a contemporary study of cyborgs (Cyborgology) is that this cybernetic organism is multiplying and seems to be everywhere. In the Cyborg Handbook
they are classified as follows (Gray et al. 1995, 14):

- Mega-cyborg: large-scale entities that include infantry using exoskeletons giant human-machine systems that serve as weapons similar to
those that appear in Star Wars, even including political bodies that span the world or the galaxy.

- Semi-cyborg: organisms that are intermittently cyborgs, such as dialysis semi-industrial 0 ties that are part of the world of the econ the times.

Fig. 11. Performance at the Movistar Loom Festival 2018. Photo by Oriol Segon Torra

Among the current debates, we should highlight the bioethical tone surround-In conclusion, the Transpecies Society is a laboratory in which hybrid pracing those who self-design and the operations on their bodies. This tone was Manel, Moon, and Neil made us ask: volved in surgical operations to design themselves? is it right to participate in a self-design project whose purpose is to improve or increase cognitive and perceptual abilities?

only affects the body but also altersidentity? How can bodies that are no longer a collection of ordered organs or which
defy what we call an organism be ethically judged? (Deleuze and Guattari

TEMES DE DISSENY \#35 tices are promoted for designing and manufacturing organs. Through thes practices, the designer is not characterized by being able to speculate about fers fore, the Transpecies Society's methodology shall be open: software codes, ecome visible and multiplied.

PICTORIAL (a) experience in which the divergent and provocative uses of cybernetics have
- Multi-cyborg: combinations of various types of cyborgs,

-

- Proto-cyborg: a cyborg lacking a complete hybrid embodiment.

- Ultra-cyborg: an enhanced cybernetic organism such as a soldier or an

th. acyby that is increasing layer after layer or prosthesis to restore a lost limb.

Meta-cyborg: a cyborg society despite its citizens not being cyborgs.

( in which an output can be part of a future input. In other words. feedback is the property of being able to adjust future behavior by taking into account past actions (Wiener 1988, 33).

3. Addressing the This ambiguity cons of the pros thesis not merely substituting an imperfection or deficiency; rather, the prosthesis is the an expansion of the body (Preciado 2011, 153).

4. Debates about the oneness betwe However, we will keep with cybernetics and assume a unified conception in which it is coherent to believe that the mind is machines, and physical and social environment (Capra 1998 71) (Damasio 2017, 337).

5. We should mention that there is a latent danger in the customization found sumers actors only to awaken their desires, thereby increasing that would make us more than ever part of the code and visual language that feeds back into the capitalist market system (Zafra

T. AFANADOR / J. PARÉS
- Neo-cyborg it has the exthe in Industry 4.0. The business sector can exploit it by making con-
REFERENCES 作

. humano. Barcelona: Destino. (2002. ¿Cómo hacerse un cuerpo sin or-

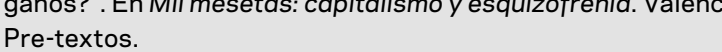
Dubberly, Hugh and Paul Pangaro. 2015. "Cybernetics and Design: Conver-
sations for Action". Cybernetics and Human Knowing 22(2-3):

73-82.
Eagleman, David. 2015. "Can we create new senses for humans?." Filmed

Franchi, Stefano and Güven Güzeldere. 1996. "Machinations of the Mird Cybernetics and Articicial intengence from Automata to cyborg". In Mechanical Bodies, Computational Minds: artificial intelligence from automata to cyborg, edited by Stef
Güzeldere, 15-149. Cambridge: MIT Press.

Gray, Hables, Chris, Mentor, Steven \& Figueroa-Sarriera, Heidi. 1995. "Cyborgology: Constructing the Knowledge of Cybernetic Organisms". York: Routledge.

Haraway, Donna. 1995, Ciencia, cyborg y mujeres: I r reinvención de la natuMadrid: Cátedra

Murray, Soraya. 2008. "Cibernated Aesthetics: Lee Bul and the Body Transfigured". Journal of Performance and Art 30(2): 38-50.

Preciado, Paul, B. 2011. Manifiesto Contrasexual. Barcelona: Anagrama. society. Boston: Da Capo teclean. Málaga: Páginas de espuma.

\section{ind
sw
aw
in-}




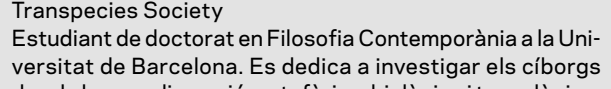

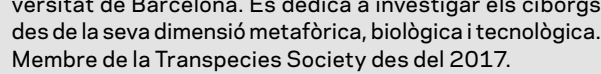
Transpecies Society
Graduada an Dissenny Ifinalista del concurus Reshapel i iestudiaint dienginineria

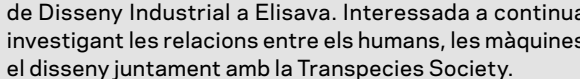

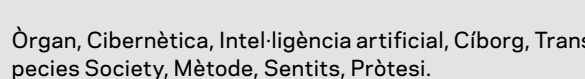

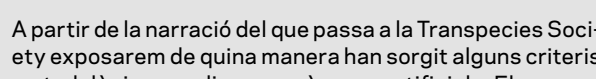

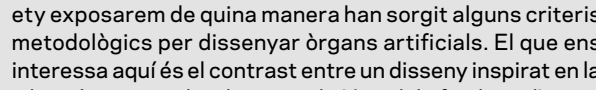

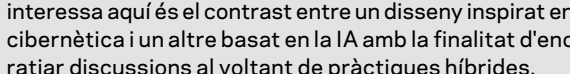

disseny dòrgans a la Transpecies Societ): pràctiques hibrides entre cibernètica $i$ intel-ligència artificial

\section{Traducció al Català}

El disseny d'örgans cibernètics no té gaire referents actuals en els quals inspirar-se. No Aquest sentit
obstant això, considerem que els membres de la Transpecies Society, que incorporen
orella alhora els organs al seu treball per tal de revelar nous estimuls de l'entorn, estan jugant amb

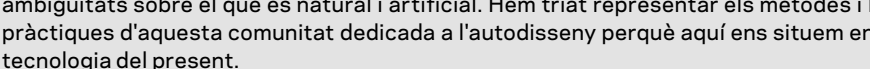

Fig. 1. Transpecies Societty és una associació que dona veu a identitats no humanes.
Aquesta societat va néixer el desembre del 2017 i iestà situada al barri del Poble Sec

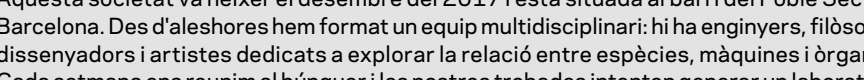

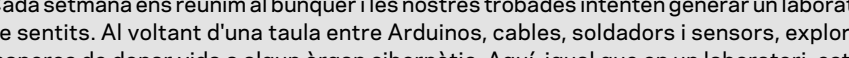
disposats a experimentar. Durante els laboratoris de sentits pot semblar que la nosträ

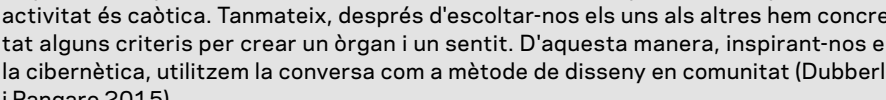

Fig. 2i 3. Considerem un ciborg [1] tot aquell qui vulgui crear òrgans cibernètics per
inventar unnou sentiti o input. El cami ique recorre aquesth hibrid entre màquina i organ co-

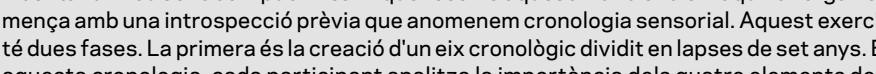

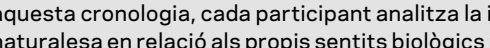

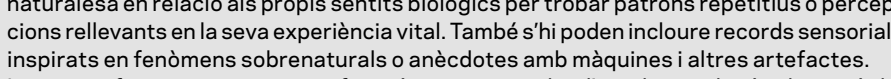

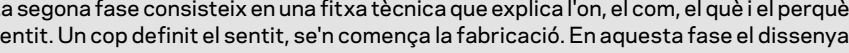

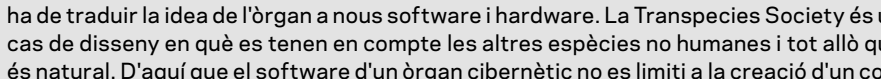

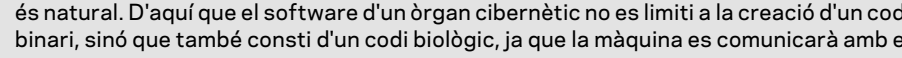

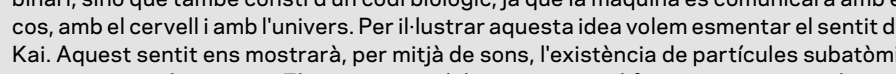

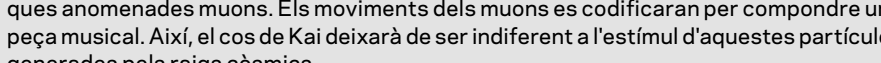

(A. 4 .

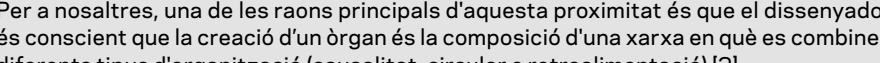
diferents tipus ''organitzaciól (causalitat, circular oretroalimentació) [2]

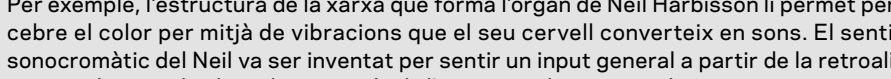

TEMES DE DISSENY \#35

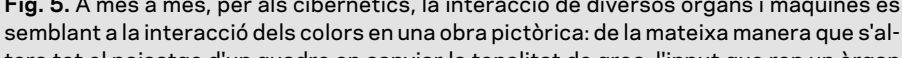

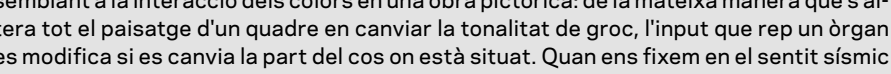

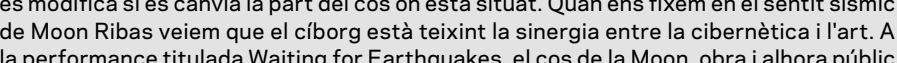
del batec de la terra, dansa els terratrèmols en temps reali els seus moviments són Fig. 6 i.7. Trobem un altre simile entre les pràctiques de l'art ila ciberrètica en la similitud
que hi ha entre els diferents prototipus d'un organ i l'escriptura d'un poema, ja que l'es criptura del codid d'un òrgan canvia o passa per tantes fases com la d'un poema. Aques

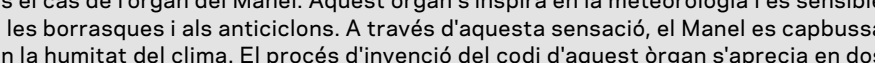

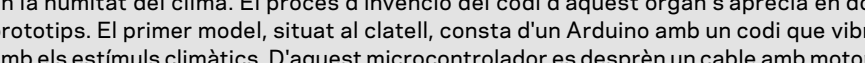
amb els estimuls climàtics. D'aquest microcontrolador es desprèn nun cable amb motor
que l'artista escolta amb el seu os temporal. El segogn prototoip són unes aletes. EI Mane

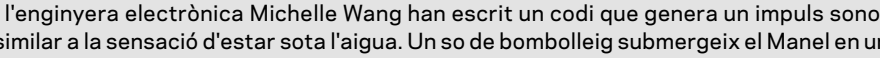

Fig. 8. Abans dimplantar un òrgan es fabriquen unes protesis anomenades exosentits
31. Aquests exosentitits són el hardware del ciborg. Un cop desenvolupat el hardware

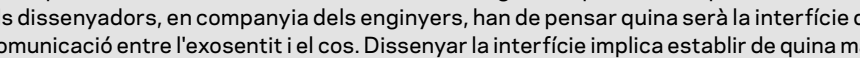

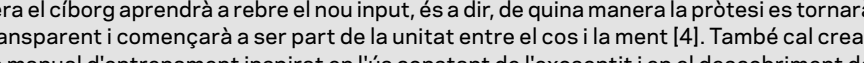

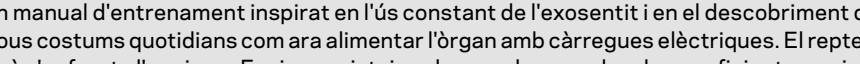
Capaç d'estalviar el consum d'energia per augmentar la la durabilitatat del funcionamentent do exosentit. I, en el futur, serà arribar a desenvolupar un òrgan que es puguia alimentar

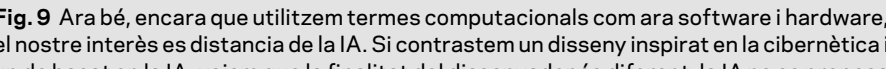
unde basat en la I A, veiem que la finalitat del dissenyador és different: I I I A no es proposa

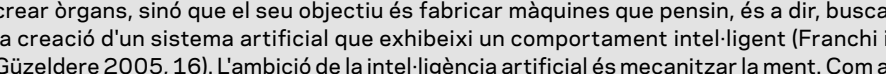
consequiuencia, el seu procodedimenten el moment de dissenyar se centra en programes aue
simulenel funcionament del cervell. Sinoés aixi, estem davant del disseny de logaritmes
PICTORIAL
150

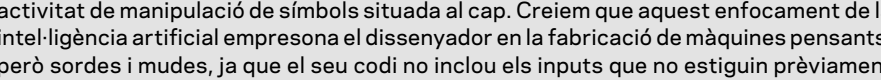

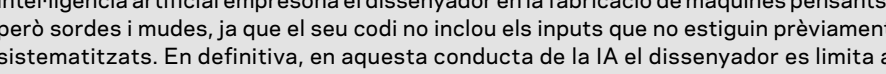

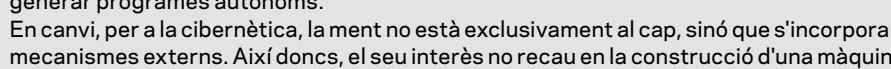

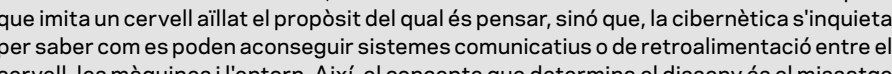

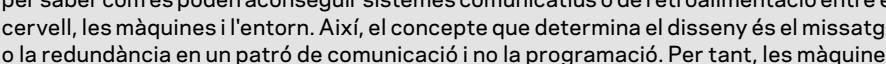

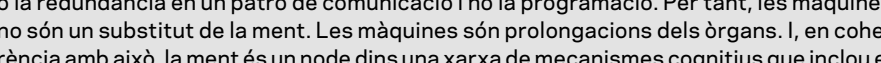

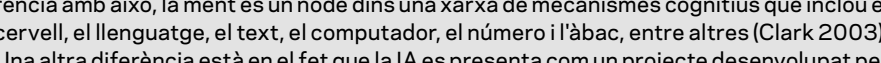

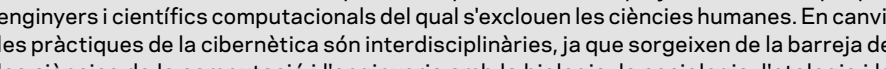

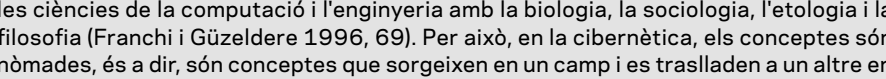
que canviene el seu significat.

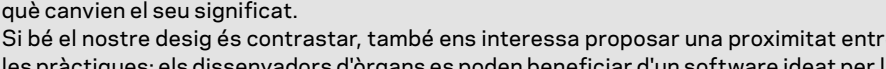

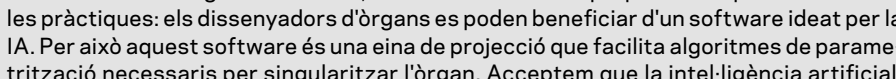

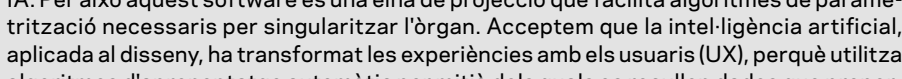
goritmes d'aprenentatatge autom
cinnen informacio personalitzada

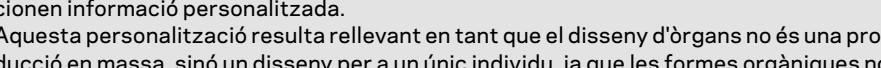

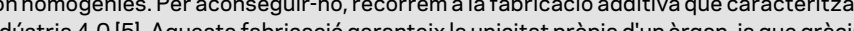

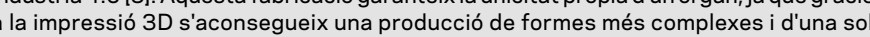

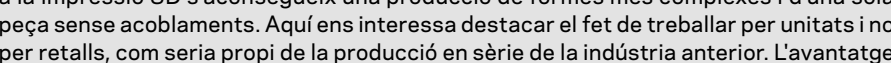

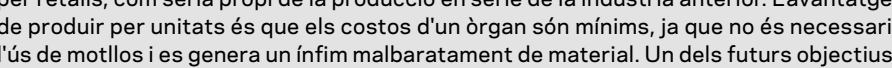

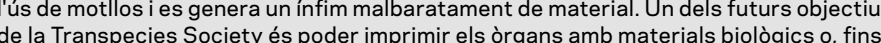

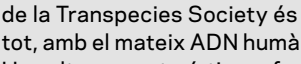

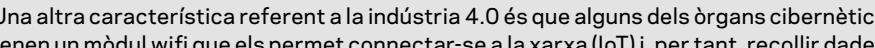

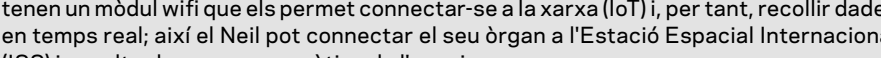

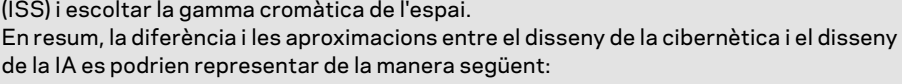

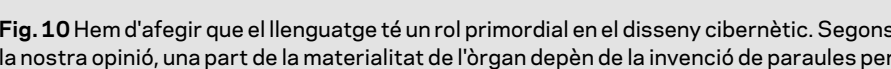

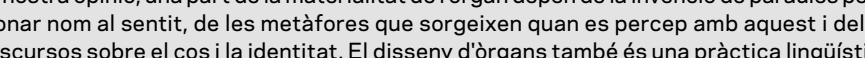

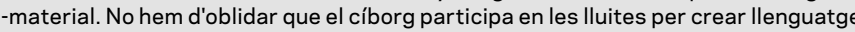

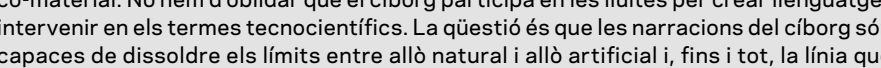

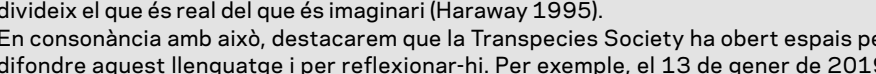

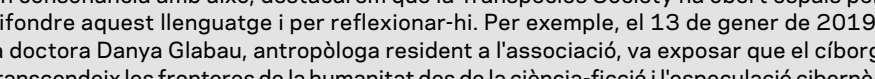

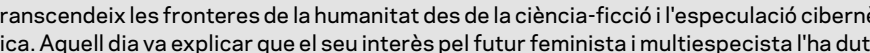

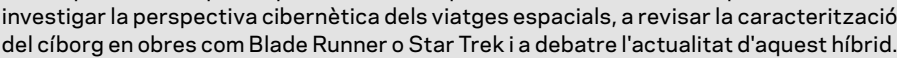

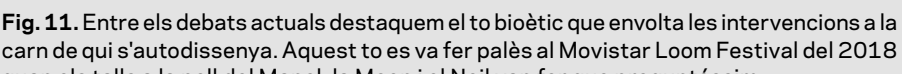

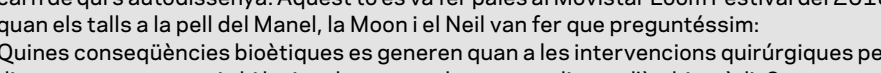

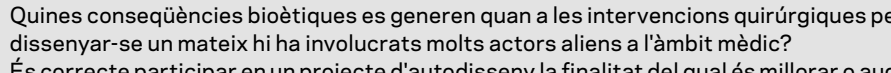

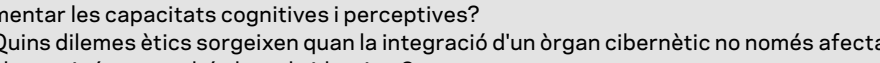

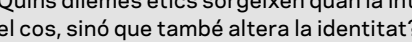

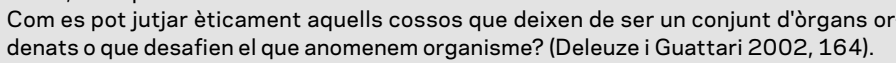
En conclusio, la Transpecies Society és un laboratori on simpulsen pràctiques hibrides

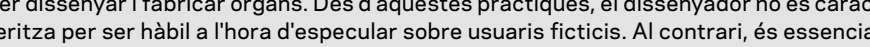

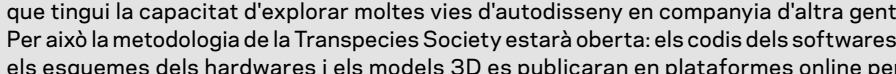

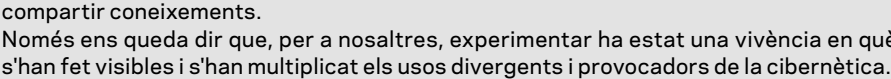

NOTES ALPEU

1.Per entendre què ès un ciborg ens remetem a la definicicío original de Cyborg and

T. AFANADOR / J. PARÉS

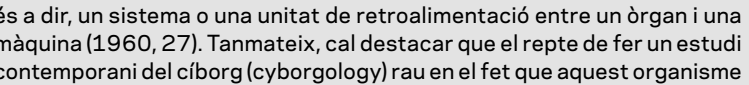
cibernètic s'stà multiplicant i isembla que és per tot arreut El IC Cyborg

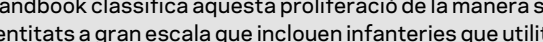
gegants sistemes humà-màquina que funcionen com a armes, similars als
que apareixen a Star Wars, ifins i tot, cossos politics que s'estenen pel món oper la agalàxia.
organismes que són

olisi connectats a una mànuinat trenta ciborgs, com els pacients de di-

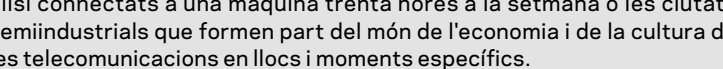

. Multicisorgs. són combinacions de diversos tipus de ciborg.

- Neociborg: exteriorment té la la forma diun cíborg, perà li li cal un un ibtegració completa Imb la protesi.

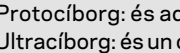

Hiperciborg extraon cor hes.

forma improvisada va afegint parts a un cos cada cop més gros. Metaciborgre restaurarar un mem membre ererdut.

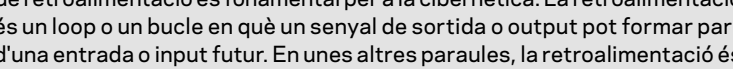
ciborg: la pròtesi no es el desenvoluupament d'un òrgan viun que genera una expannsió del cos (Preciado 2011,153). la lantitat entre cervell iment. Tanmateix. coherent creure que la mentes es relaciona amb liorganisisme completer format pel cos, el cervell, les màquines i I'ambient fisici i social (Capra 1998, 71 )
(Damasio 2017, 337).

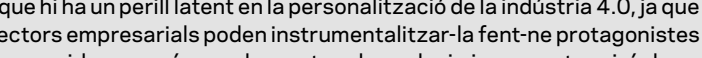
els consumidors només per despertar el seu desigi iaugmentar aixiel sos
benefici amb un cost menor. Per tant, hi hauria una homogenenitzacióm mas 作 farien més que mai part del codid idel llenguatge visu
el sistema del mercat capitalista (Zafra 2013, 126).

REFERÈNCIES 
T. Afanador y J. Parés

El diseño de órganos en la Transpecies Society:

prácticas hibridas entre cibernética inteligencia artificial

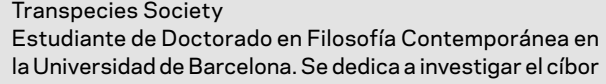

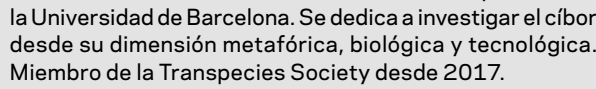
Transpecies Society
Graduadaen Diseño con el proyecto The cyborg hand (fi-
nalista del concurso Reshapel y estudiante de Ingenierí

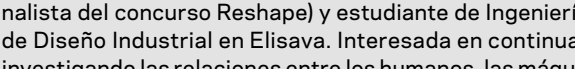
investigando las relaciones entre los humanoss, las máq
nas yel diseñojunto con la Transpecies Society Organo, Cibernética, Inteligencia Artificial, Ciborg,
Transpecies Society, Método, Sentidos, Prótesis.

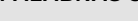

RESUMEN A partir de la narración de lo que sucede en la Transpecies
Society vamosos a exponere cómon ana surrido ciertos criterios metodológicos parae el diseño de órganos artificiciles.
Lo que nos interesa aqui es el contraste entre un diseño inspirado en la cibernética y otro basado en la 1 c con
fin de alentar discusiones en torno a prácticas hibridas.
Traducción al Castellano

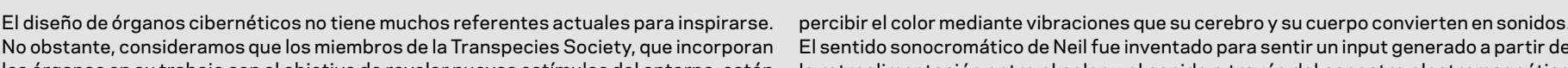
los órganos en sutrabajo con el objetivo de revelar nuevos estimulos delentorno, están métodosos yrácticias de estatacon
en la tecnologia del presente.

Fig. 1. Transpecies Society es una asociación que da voz a identidades no humanas.
Esta sociedad nació en diciembre de 2017 y está ubicada en el barrio de Poble Sec d. Barcelona. Desdde entonces hemos conformadoun equipo multidisisciplinarir hay ingenie-
ros, filosofos, diseñadores y artistas dedicados a explorar la relación entre especies, máquinas y órganos.
Semananalmente nos reunimos en el búnker y nuestros encuentros intentan generar un sensores exploramos cómo dar vida a algún órgano cibernéticic. Alli, al igual que en
laboratorio, estamos dispuestos a experimentar. Durante los laboratorios de senticas laboratorio, estamos dispuestos a experimentar. Durante los laboratorios de sentidos
puede parecer que nuestro hacer es caótico. Sin embargo, despuess de essucharnos a otros hemos concretado ciertos criterios para crear un órgano y y sentido. De es
modo, inspirándonos en la cibernética, usamos la conversación como método de diseñ

Fig. 2 y. Consideramos un ciborg [1] a todo aquel que desee crear órganos cibernéticos
para añadir un nuevo sentido o input. La senda que recorre este hibrido entre máquin

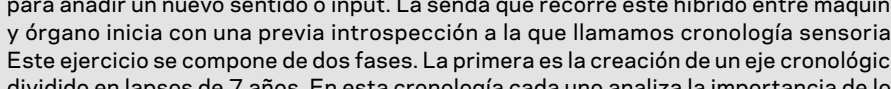

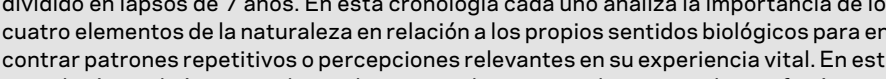

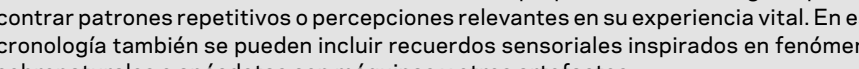

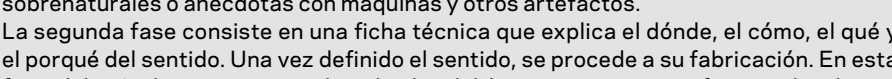
fase el diseñador tiene que traducir la idea ale organo a un nuevo software y hardware

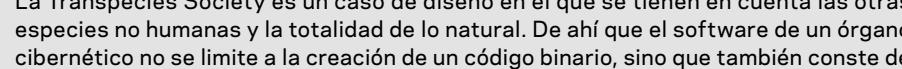

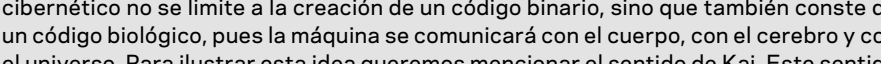

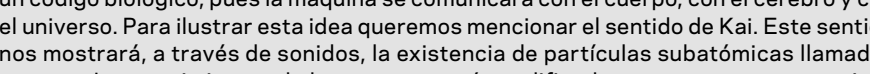
muones. Los movimientos de los muones serán codificados para componer una pieza
musical. Asi, el cuerpo de Kai dejará de ser indifierente al estímulo de estas particulas generadas por los rayos cósmicos.

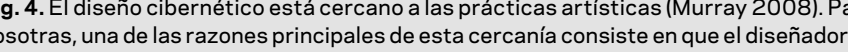

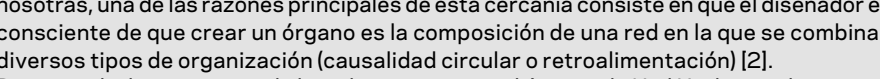

TEMES DE DISSENY \#35

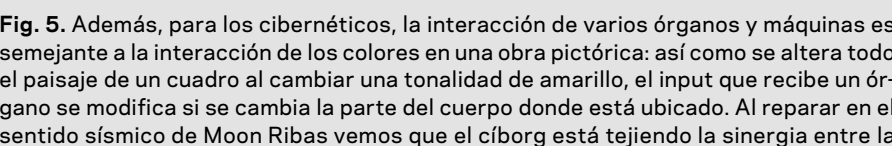
ciberńtica y el arte. En la performance llamada Waiting for Earthquakess, el cuereo de mplantado en sus pies.

Fig. 6 y 7 . Otro simil entre las prácticas del artey la cibernética lo encontramos en la
semejanza que existe entre los diferentes prototipos de un orrgano y la escritura de un

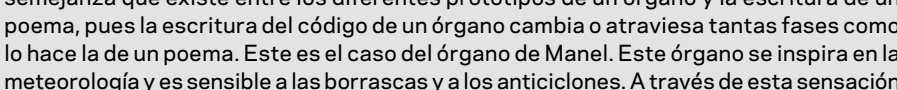
meteorología ves sensiblea a las borrascasas y a los anticiclones. A través de esta sensación a gano se apreciaen dos protototipos. El I primer modelo, ubicado en la nuca, consta de un Adeuino con un código que vibra con los estimulos climáticos. De este microcontrolaador

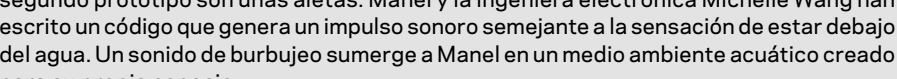

Fig. 8. Antes de que un órgano sea implantado se fabrican unas prótesis llamadas exo-
sentidos 31 . Estos exosentidos son el harddare del ciborg. Una vez desarrollado ol

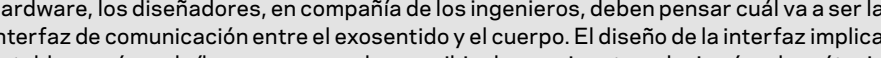
sevablecer cómo el ciborg va a aprender a recibiri l Inuevoinput, es deciri, cómo la prótetesis del exosesntido y y en el descubriminiento de nuevas costumbres cotidianas com calimentar elórgano de cargas eléctricas. El reto al que se enfrenta el lingeniero Fenix consiste en

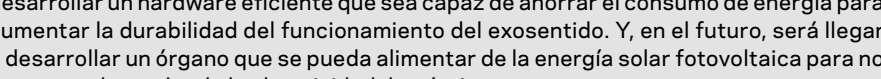

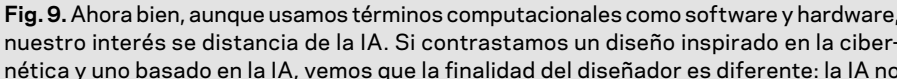
152

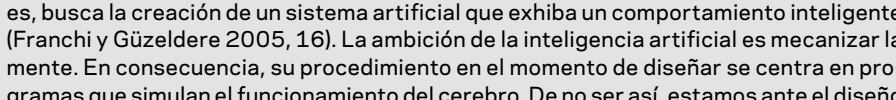
pensamiento humano e equivale a auna actividad de manipulaciónd de simbolos ubicada en

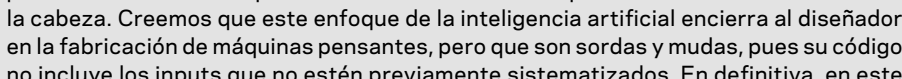

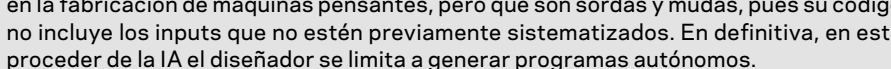
En cambio, para la a cibernéntica la la mente no e está exclusivivamente en la cabeza, sino que se incorporae en mecanismos externos. Su interés no recae entonces en la construcción
de una máauina que inita a un cerebro aislado cuyo proposito es pensar. Antes bier. le una máquina que imita a un cerebro aislado cuyo propósito es pensar. Antes bien
la cibernética se inquieta por saber cómo lograr s sistemas comunicativos o de retroal-

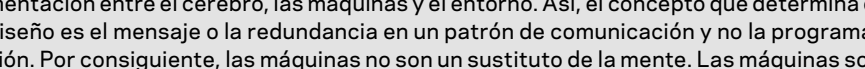
prolongaciones de los órganos. Y, en coherencia con esto, la mente es un nododo dentto de una red de mecanismos cognitivos que incluye el cerebro, el lenguaje, el texto, el
computador, el número y y el ábaco, entre otros (Clark 2003). Otra diferenciaie stáen que lal As se presentac comoun proyecto desarrollado por ingenie-
ros ycientfficos computacionales, en el que se excluyen las ciencias humanas. En cambio.

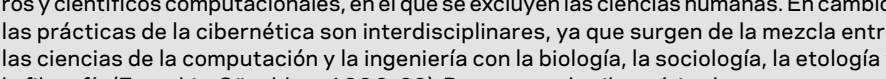
las ciencias de la computación y la ingenenieria con la biologia, la socioiologia, la etologiay
la filosoffia que cambian su significado.

las prácticas: los diseñadores de órgambiess nos interesesa proponer una proximidad entro " lal|A. Por esso este software es una herramienta de proyección eue facilita a algoritmos de
parametrización necesarios para singularizar el órgano. Aceptamos que la inteligencia

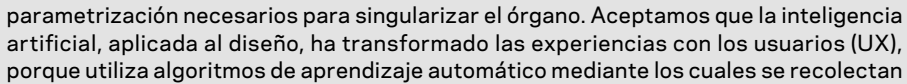
porque utiliza algoritmos de aprendizaje automaticcone

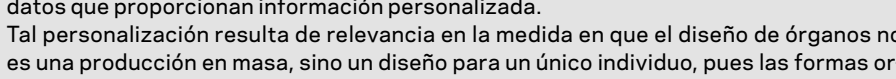
génicas no son homogéneas. Para lograr esto, recurrimos a la a fabricación aditiva que

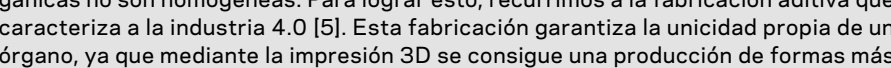
brgano, ya que mediante la limpresión $3 \mathrm{D}$ se consigue una producción de formas más
complejas y y una sola pieza sin ensamblajes. Aqui nos interesa destacar el hecho de

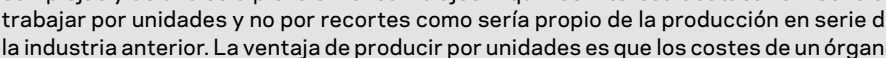

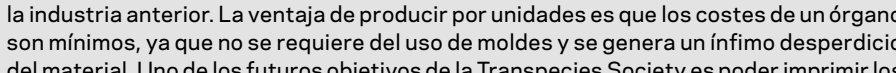
.

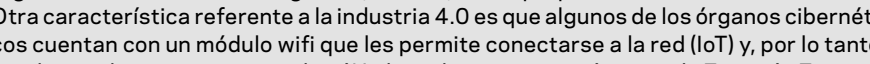

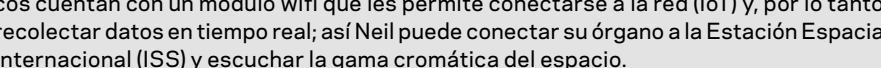

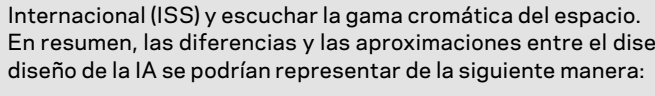

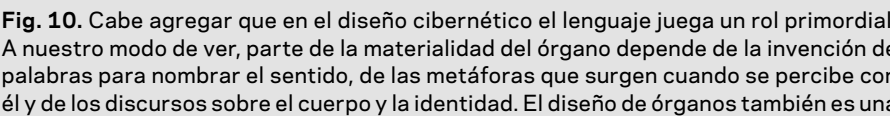

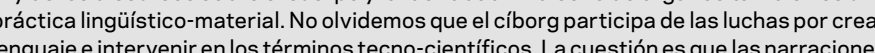

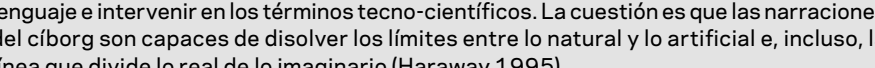
En consonancia con esto, resaltamos que la Transpecies Society ha abierto espacios
para la dafifusión y la reflexión de este lenguaje. Por ejemplo, el 13 de enero de 2019 la

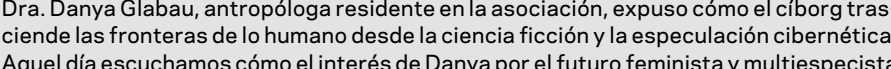

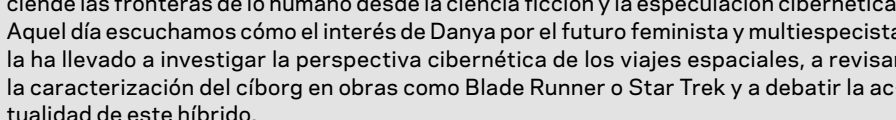
la caracterización del cibor
tualidad de este hibrido.

Fig. 11. Entre los debates actuales resaltamos el tono bioético que envuelve las inter-
venciones en la carne de quien se autodiseñá. Este tono lo evidenciamos en el Movistar Loom Festival del 2018 cuando los cortes en la piel de Manel, Moon y Neil nos hicieror

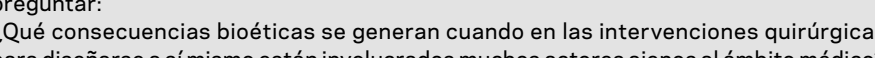
para diseñarse a si mismo están involucrados muchos actorese ajenos al ámbitom médico?
¿Es correcto participar en un provecto de autodiseño cuy fin es mejorar o aumentar la capacidades cognitivasy perceptuales? I cuerpo, sino que también altera la identidar?

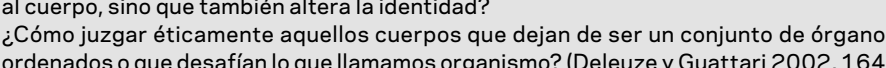

En conclusión, la Transpecies Society es un laboratorio donde se impulsan prácticas no se caracterizza por ser habbil para espececular sobre usuarios ficticticios. Al contrario, es

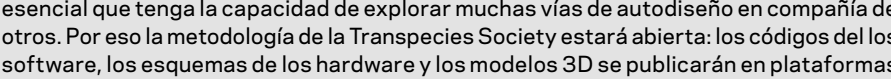

T. AFANADOR / J. PARÉS
NOTAS AL PIE

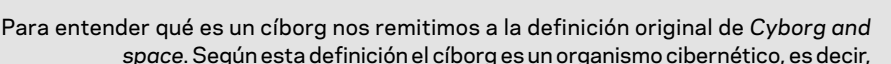
un sistema o unidadd de retroalimentación entre un órgano y una maquiura
$1960,27)$. Sin embargo, hay que resaltar que el reto de hacer un estudio

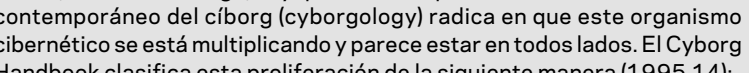

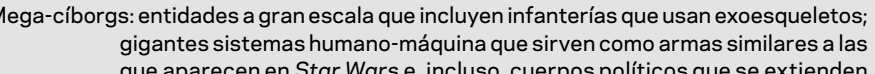
que aparecen en Star Wars
por el mundo o por la galaxia

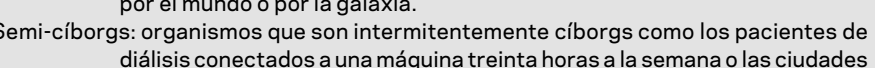

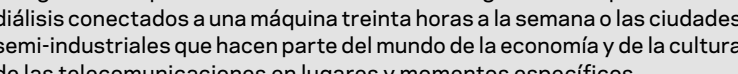

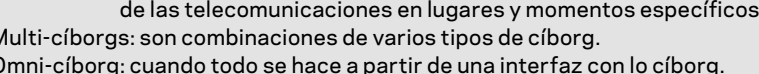

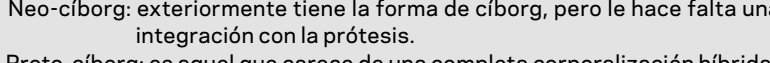

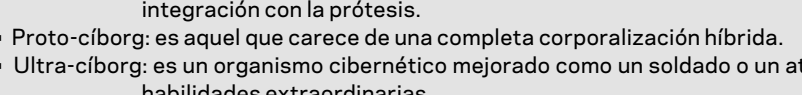

iper-ciborg: es una corrorts

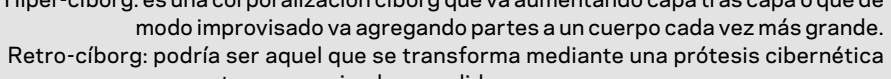
Mara restaurar un miembro perdido.
Meta-cíbrge es una sociedad ciborg a pesar de que sus ciudadanos no 0 sean.

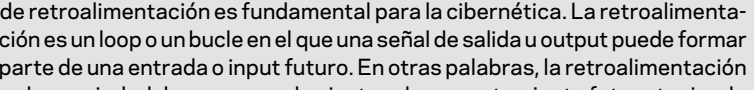
es la propiedad de ser capaz de ajustar el comportamiento futuruo teniendo en cuenta actuaciones del pasado (Wiener 1988, 33). consiste en que laprótesis no sereduce a suplir una imperfección o carenciaz
antes bien, la prótesis es la modificación y el desarrollo de un orrgano vivo del cuerpo (Preciado 2011,15

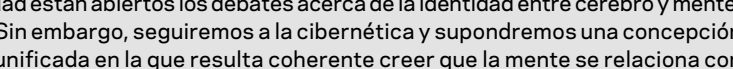
el organismo completo formado por el cuerpo, el cerebro, las máquinas y el

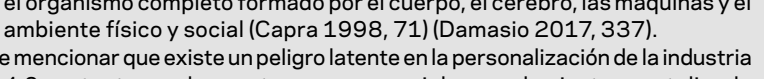

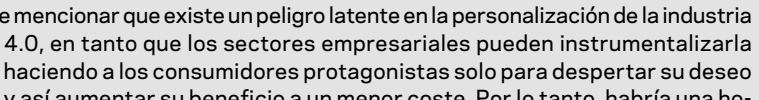

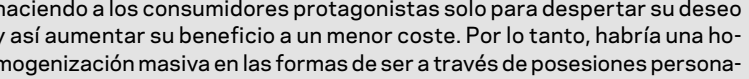

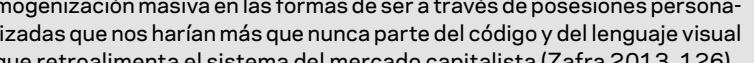
REFERENCIAS

Ver listado completo de referencias en la página 149 